\title{
Das Deutsche Arktisbüro: Wissenstransfer und Informationsaustauch für zukunftsorientierte und nachhaltige Entscheidungen für die Arktis
}

\author{
Volker Rachold \\ Deutsches Arktisbüro, Alfred-Wegener-Institut Helmholtz-Zentrum für Polar- und Meeresforschung, \\ Telegrafenberg A45, 14473 Potsdam, Deutschland
}

Correspondence: Volker Rachold (volker.rachold@arctic-office.de)

Published: 20 May 2021

Kurzfassung. Das Deutsche Arktisbüro am AlfredWegener-Institut, Helmholtz-Zentrum für Polar- und Meeresforschung (AWI) versteht sich als Informations- und Kooperationsplattform für deutsche Arktis-Akteure aus Wissenschaft, Politik und Wirtschaft und dient dem AWI in erster Linie als Instrument für den Wissenstransfer. Das Büro fungiert als einheitlicher Ansprechpartner für Bundesministerien, Behörden etc. und Anlaufstelle für die deutsche Wirtschaft. Es berät die Bundesregierung durch wissenschaftliche Begleitung von Positionen zu Arktisfragen und stärkt die deutsche Beteiligung an den wissenschaftlichen Aktivitäten des Arktischen Rats. Es vermittelt konsistent aufbereitete Informationen aus Arktisforschung und -politik und liefert Impulse für die Wissenschaft durch Vermittlung politikrelevanter Fragen an die entsprechenden wissenschaftlichen Institutionen. Die Konzeption des an der Forschungsstelle Potsdam des AWI angesiedelten Büros erfolgte in enger Abstimmung mit den relevanten Bundesministerien, insbesondere mit dem für die deutsche Arktispolitik federführenden Auswärtigen Amt (AA).

Abstract. The German Arctic Office at the Alfred Wegener Institute Helmholtz Centre for Polar and Marine Research (AWI) serves as an information and cooperation platform at the interface between science and policy and contributes to AWI's knowledge transfer. The office supports the German Federal Ministries concerned with Arctic affairs by fostering dialogue and information exchange and providing knowledge to support future-oriented and sustainable Arctic decision making.

\section{Arktisdialog}

Das zentrale Instrument für den Informationsaustausch zwischen Arktisforschung und Arktispolitik ist der vom Arktisbüro organisierte Arktisdialog. Die halbjährlich stattfindenden Informationsveranstaltungen behandeln aktuelle Fragen der deutschen und internationalen Arktisforschung und -politik. An den Veranstaltungen nehmen Vertreter von sieben Bundesministerien und den zugehörigen Bundesbehörden, Stiftungen und Nichtregierungsorganisation sowie alle in der Arktisforschung engagierten wissenschaftlichen Institute teil. Durch den Arktisdialog werden den an Arktisfragen interessierten Bundesministerien Wissen, Erfahrung und internationale Kontakte gezielt zur Verfügung gestellt. Gleichzeitig haben die Veranstaltungen auch zu einem verbesserten Dialog zwischen den Ministerien geführt und so zu der Verabschiedung der Leitlinien der deutschen Arktispolitik (https://www.auswaertiges-amt.de/de/aussenpolitik/themen/ internationales-recht/einzelfragen/arktis-leitlinien/2239800, letzter Zugriff: 11. Februar 2021) maßgeblich beigetragen.

\section{Zweite Arktische Wissenschaftsministerkonferenz}

Die internationale wissenschaftliche Zusammenarbeit ist von entscheidender Bedeutung für die Beobachtung und das Verständnis der raschen Veränderungen, die in der Arktis ablaufen. Deutschland gehört auf dem Gebiet der Arktisforschung zu den international führenden Nationen und übernimmt internationale Verantwortung. Aufbauend auf der ersten Arktischen Wissenschaftsministerkonferenz, die im September 2016 in Washington stattfand, haben die Euro- 
päische Kommission, Finnland und Deutschland die zweite Arktische Wissenschaftsministerkonferenz (https://www. arcticscienceministerial.org/index.html, letzter Zugriff: 11 . Februar 2021) in Berlin am 25.-26. Oktober 2018 organisiert. 26 Regierungen, 6 indigene und 10 internationale Organisationen haben an der Konferenz teilgenommen, die sich über zwei Tage erstreckte. Das Arktisbüro hat das Bundesministerium für Bildung und Forschung (BMBF) bei der Vorbereitung und Durchführung der Konferenz unterstützt indem es das Programm des Arktischen Wissenschaftsforums am ersten Tag der Konferenz gestaltet hat und die wissenschaftlichen Beiträge der teilnehmenden Länder und Organisation analysiert und in einem Abschussbericht zusammengestellt hat.

\section{Arktischer Rat}

Deutschland ist im Arktischen Rat (https://www. arctic-office.de/politikberatung/arktischer-rat/, letzter Zugriff: 11. Februar 2021, https://arctic-council.org/en/, letzter Zugriff: 11. Februar 2021) als Beobachter durch das AA vertreten, das bestrebt ist, die deutschen Beiträge zu den Aktivitäten des Rats zu stärken. Insbesondere die Beteiligung an den Arbeitsgruppen und Task Forces des Arktischen Rates wurde in den letzten Jahren deutlich verbessert. Für alle Arbeitsgruppen und Task Forces wurden deutsche Experten benannt. Das Arktisbüro unterstützt das AA bei der Auswahl dieser wissenschaftlichen Experten und sorgt dafür, dass Berichte von den Sitzungen sowie Handlungsempfehlungen verfügbar gemacht werden.

\section{Publikationen}

Um das steigende Interesse an bestimmten Themenbereichen der Arktis zu bedienen, publiziert das Arktisbüro Hintergrundpapiere und FactSheets (https://www.arctic-office.de/ publikationen/, letzter Zugriff: 11. Februar 2021). Die Themenauswahl ergibt sich aus den an das Büro herangetragenen Anfragen. Die Fact Sheets liefern einen kurzen und leicht verständlichen Überblick und richten sich an Arktisinteressierte Mitarbeiter*innen von Ministerien, Behörden und wissenschaftlichen Einrichtungen sowie an Medienvertreter*innen und die allgemeine Öffentlichkeit.

\section{Informationsveranstaltungen}

Das Arktisbüro initiiert eigene nationale Arktisveranstaltungen mit Partnern aus Wissenschaft, Politik und Wirtschaft und unterstützt die Bundesregierung bei der Vorstellung der deutschen Arktisforschung und -politik bei internationalen Konferenzen. Die Zusammenarbeit mit den in Berlin ansässigen Botschaften der Arktis-Anrainer ist dabei von besonderer Bedeutung. Aus dieser Kooperation sind in den letzten Jahren etliche gemeinsame Veranstaltungen entstanden.

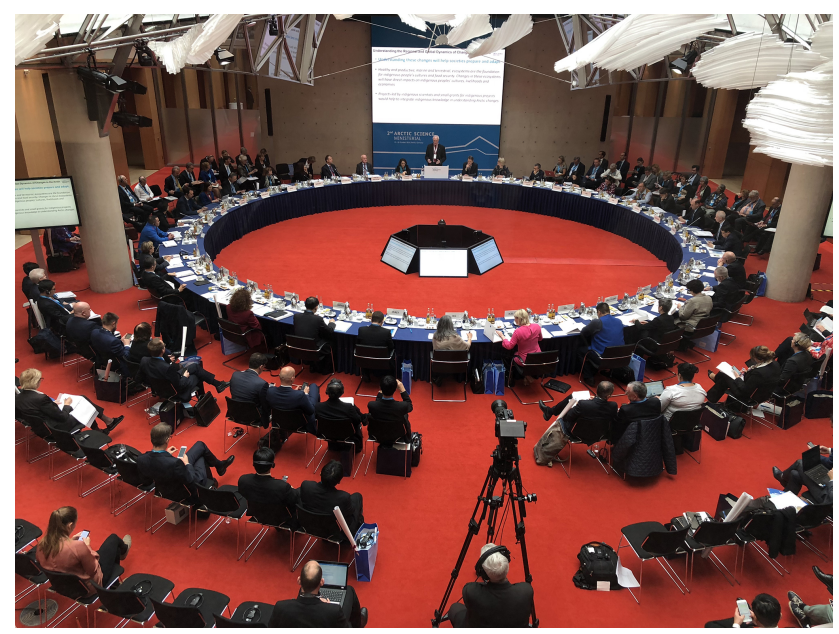

Abb. 1. Zweite Arktische Wissenschaftsministerkonferenz, Berlin, 26.-27. Oktober 2018 (Foto: Volker Rachold).

Gleichzeitig hat das Arktisbüro enge Kontakte zu den deutschen Botschaften in den Arktisstaaten etabliert, aus denen sich ebenfalls gemeinsam organisierte Veranstaltungen ergeben haben (https://www.arctic-office.de/veranstaltungen/, letzter Zugriff: 11. Februar 2021).

Mit dem Arktisbüro verfügt das AWI über ein Instrument, um politischen Entscheidungsträgern auf direktem Weg wissenschaftliche Beratung anbieten zu können. Dieser direkte Zugang zur Bundesregierung ermöglicht es dem AWI, wissenschaftliche Ergebnisse in relevante politische Entscheidungsprozesse einzubringen und so zu einer wissenschaftsbasierten deutschen Arktispolitik aktiv beizutragen.

Datenverfügbarkeit. Für diesen Artikel wurden keine Datensätze genutzt.

Interessenkonflikt. Die Autor*innen erklären, dass kein Interessenkonflikt besteht.

Begutachtung. This paper was edited by Bernhard Diekmann. 